\title{
AiMT
}

Advances in Military Technology

Vol. 15, No. 2, 2020, pp. 355-364

ISSN 1802-2308, eISSN 2533-4123

DOI 10.3849/aimt.01385

\section{Influence of Wave Shaper Position on Jet Formation and Penetration Depth}

\author{
V. Minh Do ${ }^{1}$, M. Thai Le ${ }^{1}$, X. Son Bui ${ }^{1 *}$, H. Nguyen Pham ${ }^{1}$ \\ and P. Linh Nguyen ${ }^{2}$ \\ ${ }^{1}$ Faculty of Weapons, Le Quy Don Technical University, Hanoi, Vietnam \\ ${ }^{2}$ The General Department of Defense Industry, Hanoi, Vietnam
}

The manuscript was received on 21 January 2020 and was accepted after revision for publication as research paper on 26 September 2020.

\begin{abstract}
:
The article presents the influence of the wave shaper position on the jet formation and penetration depth of a shaped charge, which uses the liner of the PG-7VM warhead by numerical method and experimental method. The results show that, when moving the plexiglass wave shaper position towards the liner apex, the jet tip velocity and the jet velocity gradient increase. In addition, the results of the study also pointed out the existence of the wave shaper position at which the penetration depth is greatest. They indicate a match between the simulation and experimental results. The differences between simulated and experimental penetration depth are less than 15\%. The results of the study are the basis for the evaluation of the influence of the wave shaper position on the liner collapse and the reasonable use of the wave shaper in the structure of the shaped charge.
\end{abstract}

\section{Keywords:}

jet formation, jet velocity, penetration depth, shaped charge, wave shaper

\section{Introduction}

During the explosion process, it is possible to control the detonation wave to focus the energy of the charge that makes up the line, region, and surface where there are extremely high density and pressure. The phenomenon of explosion energy concentration is widely applied in both the military and civilian areas. In the military field, it may be encountered in the structure of a shaped charge and an explosively formed penetrator. One of the detonation wave control schemes to increase the penetration depth of the shaped charge is the use of a wave shaper in its structure. When using the wave shap-

\footnotetext{
* Corresponding author: Department of Munitions, Le Quy Don Technical University, Hoang Quoc Viet 236, Hanoi, Vietnam. Phone: +84948918486, E-mail: buixuanson.mta@ gmail.com
} 
er, its position has an important influence on the penetration depth of the shaped charge. This conclusion has been presented in several documents. However, the quantitative research of the influence of the wave shaper position on the penetration depth of the shaped charge given in some documents was not fully evaluated [1-5].

Marina [1] proposed the method of calculating the penetration depth of the shaped charge taking into account the effect of the sub-initiation point ( $T$ point in Fig. 1). The calculated result of this method shows that the penetration depth increases when the wave shaper diameter is constant if the position of the sub-initiation point moves to the top of the liner. However, in practice, the wave shaper position is not placed at the top of the liner. It suggests that the wave shaper position at the top of the liner is not the optimal position.

Zhu et al. [2] researched the process of controlling the Mach wave surface in the structure of the shaped charge with the use of the wave shaper. Results indicate that when the wave shaper is embedded in charge, the pressure behind the Mach wave can be controlled by changing the wave shaper diameter and the distance between the wave shaper and the liner.

$\mathrm{Zu}$ et al. [3] investigated the detonation wave propagation in the shaped charge using the wave shaper. The calculated result indicates that the three types of detonation wave surfaces, such as conical detonation wave, spherical detonation wave, and flat explosive wave can be formed in the main charge by the change of the wave shaper thickness. At the same time, the flat wave surface is better than two other wave surfaces in increasing the length - diameter ratio of the explosively-formed projectiles and keeping the nose of the warhead intact.

Guo et al. [4] studied the effect of the wave shaper on jet parameters and penetration depth. The research results show that jet tip velocity, and jet velocity gradient increase if the wave shaper is used.

Hussain [5] and Guo et al. [4] asserted that the angle between the detonation wave surface and liner generatrix when using the wave shaper is smaller than in the case of not using the wave shaper. Therefore, the pressure after the wave surface impacting the liner increases when using the wave shaper.

The purpose of the article is to study the effect of the wave shaper position on the jet formation and penetration depth with the Ansys Autodyn 2D software. The process of the detonation wave propagation, jet parameters such as kinetic energy, the jet velocity gradient and jet tip velocity have been presented in this study. Besides, experiments were also conducted to determine the penetration depth of the shaped charge using the liner of the PG-7VM warhead with different positions of the wave shaper. The experimental results were analysed and compared with simulated results.

\section{Numerical Method and Material Model}

Based on [6, 7], the simulation problem consists of the process of forming the jet and the impact of the jet into the steel target. The jet parameters obtained in the simulation of the jet formation are the inputs for the simulation of the impact of the jet into the steel target. The material of the shaped charge components and the material models are given in Tab. 1.

The material parameters of the liner, the case, the para-charge, and the wave shaper are taken from the software library [8]. Material parameters of the main charge are presented in [9]. 
Tab. 1 Equation of state and material models

\begin{tabular}{|l|c|c|c|c|}
\hline \multicolumn{1}{|c|}{ Part } & Material & $\begin{array}{c}\text { Equation of } \\
\text { State }\end{array}$ & Strength Model & $\begin{array}{c}\text { Failure } \\
\text { Model }\end{array}$ \\
\hline Liner & CU-OFHC & Shock & Steinberg-Guinan & - \\
\hline Case & Polyethylene & Shock & - & - \\
\hline Main charge & C4 & Lee-Tarver & von Mises & - \\
\hline Wave shaper & Plexiglass & Shock & - & - \\
\hline Para-charge & C4 & JWL & - & - \\
\hline Target & C45 Steel & Shock & Johnson-Cook & Johnson-Cook \\
\hline
\end{tabular}

The shaped charge model used in the simulation is based on the structure of the PG-7VM warhead. This model is shown in the XOY coordinate system where OX is the symmetry axis of the shaped charge, $\mathrm{T}$ is the position of the sub-initiation point, $z w s$ is the distance between the wave shaper and the top $\mathrm{O}$ of the liner.

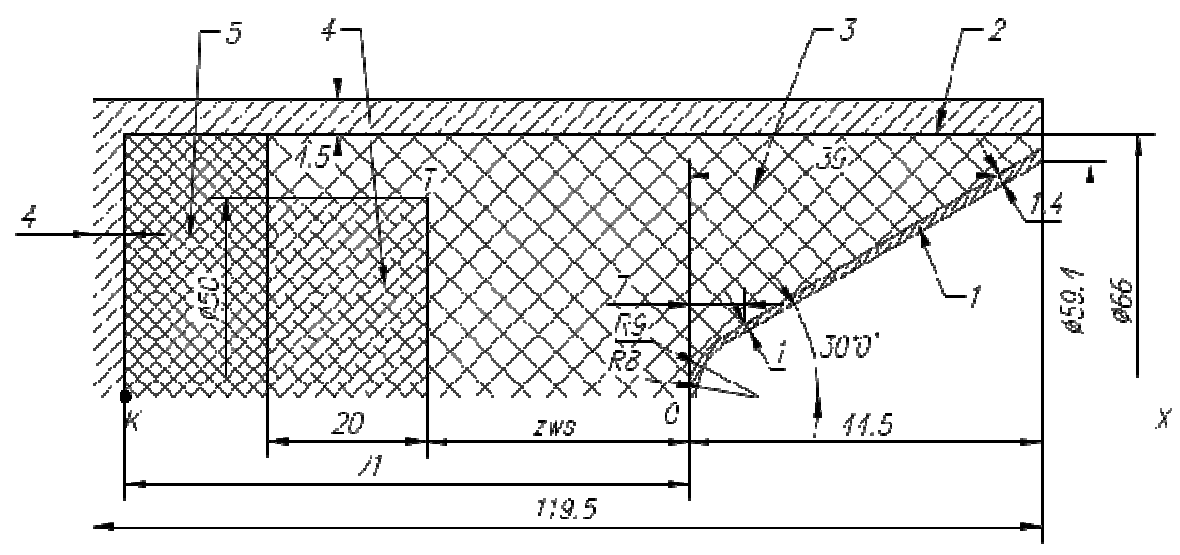

Fig. 1 The shaped charge model (in $\mathrm{mm}$ )

1 - Liner; 2 - Case; 3 - Main charge; 4 - Wave shaper; 5 - Para-charge;

$O$ - Origin of coordinates; $T$ - Sub-initiation point; $K$ - Detonation point.

The construction of simulation models on Ansys Autodyn 2D software is based on the geometric dimensions shown in Fig. 1. Due to the symmetry of the shaped charge, the multi-material Euler-2D solver is used to simulate the propagation of the detonation wave and the liner collapse $[6,7]$. In the simulation model of the process of the jet formation, the size of the square cells was selected to be $0.25 \mathrm{~mm}$. The interactions of detonation products and case at the boundary of the calculation area are not considered. The simulation time ends when the jet tip reaches a distance of twice the charge diameter from the liner base $[6,7]$. This distance is fixed during the simulation to be able to evaluate the effects of the wave shaper position on the jet formation and penetration depth. It is not the optimal standoff distance for the maximum penetration depth. The point detonation method is used in the simulation. The position of the detonation point $K$ is shown in Fig. 1. The Flowout boundary condition is applied to all computational boundaries. It allows the detonation products to expand and the case to fly out of the computation area without interacting with the 
boundaries and without affecting the collapse of the liner. The simulation model of shaped charge is shown in Fig. 2.

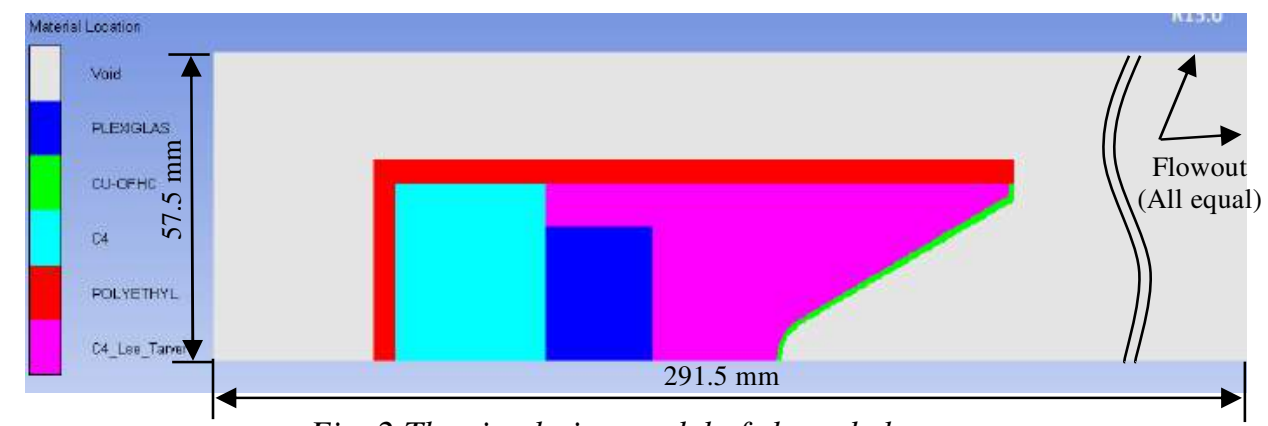

Fig. 2 The simulation model of shaped charge

In the penetration process simulation, the Lagrange grid is applied for both the jet and the target. The simulation results of the jet formation by the Euler method are used to determine the jet parameters. The jet strikes the target at a two-charge-diameter standoff distance $[6,7]$. The target material is C45 steel and the cylindrical target is $130 \mathrm{~mm}$ in diameter and $400 \mathrm{~mm}$ in length. The equation of state for the target material is shock state equation, while its strength model and failure model are the Johnson-Cook constitutive model [10]. The simulation model of the impact process between the jet and the steel target is shown in Fig. 3. Both the jet and the target parts are modeled using $0.5 \mathrm{~mm}$ rectangular elements [6]. For the target, however, the mesh size is extended in the direction of the radius from the radius of $15 \mathrm{~mm}$ to reduce the total number of elements. Because of the symmetry, the simulation was performed with half of the models of both the jet and the target.

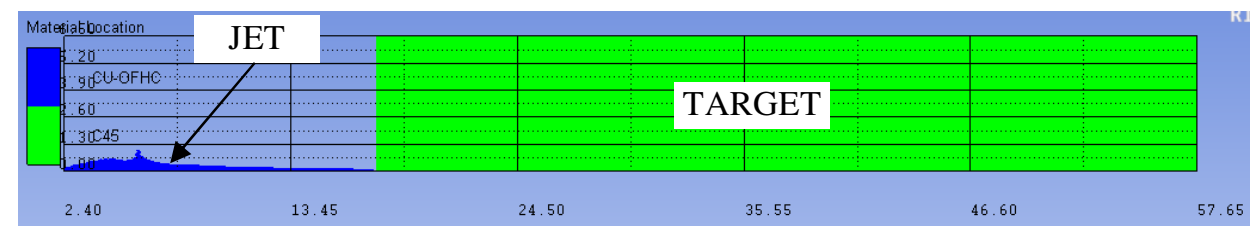

Fig.3 Simulation model of impact process between the jet and the steel target

The options are given in Tab. 2 with different positions of the wave shaper, which affect the simulation results.

Tab. 2. Different positions of wave shaper

\begin{tabular}{|c|r|r|r|r|}
\hline Options & 1 & 2 & 3 & 4 \\
\hline$z w s[\mathrm{~mm}]$ & -33 & -23 & -13 & 0 \\
\hline
\end{tabular}

\section{Experiment}

To assess the results of the simulation method, the experiments with different wave shaper positions have been carried out. The shaped charge configurations used in the experiments are based on the simulation model in previous chapter. 


\subsection{Shaped Charge and Experimental Target}

The liner used for experimental shaped charges is the liner of the PG-7VM warhead, whose dimensions are shown in Fig. 4.

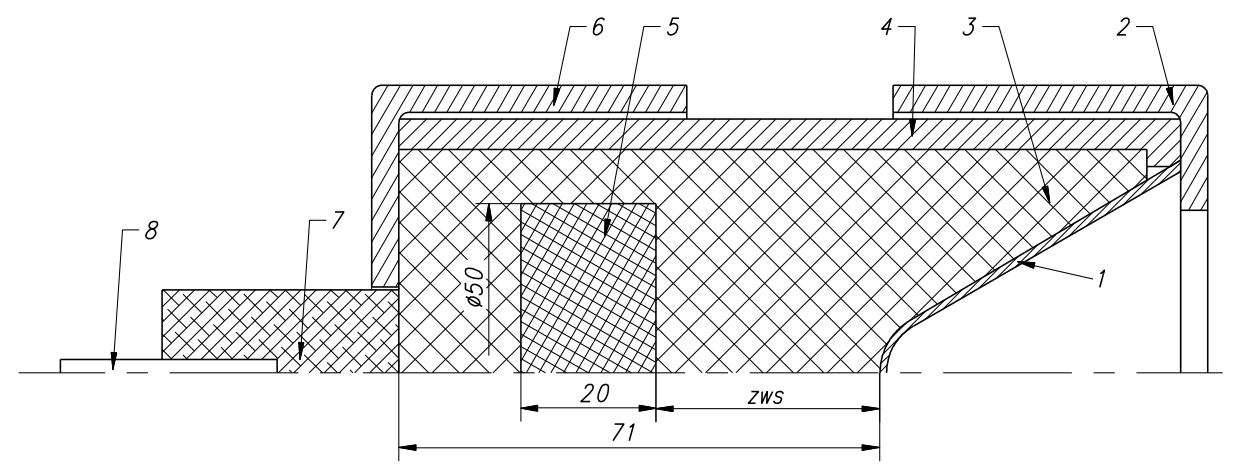

Fig. 4 Geometric model of experimental shaped charge [mm]

1 - Liner; 2 - Top cover; 3 - Explosive; 4 - Case; 5 - Wave shaper;

6 - Bottom cover; 7 - Booster; 8 - Detonator

The material of the liner is copper. The material of the covers and the case is PE. The material of the wave shaper is plexiglass. The dimensions of the cylindrical wave shaper are $50 \mathrm{~mm}$ in diameter and $20 \mathrm{~mm}$ in thickness (Fig. 5). The material of the booster is the ТГ- 50 explosive. The material of the explosive is composition C-4. The electrical detonator is used to detonate the booster. The material of target is $\mathrm{C} 45$ steel. The dimensions of the cylindrical target are $130 \mathrm{~mm}$ in diameter and $300 \mathrm{~mm}$ in length (Fig. 6).

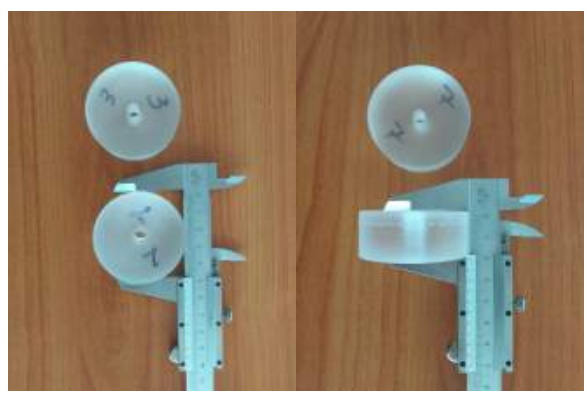

Fig. 5 Wave shaper

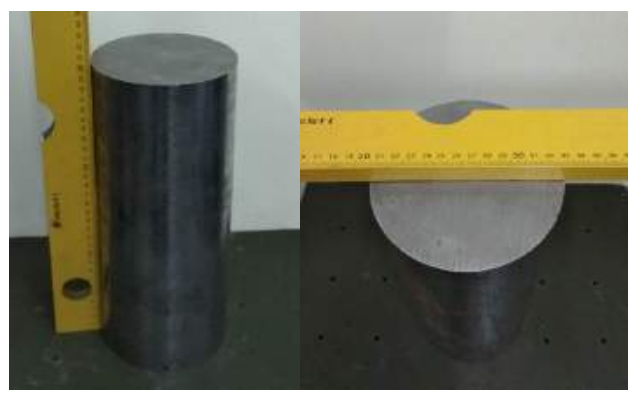

Fig. 6 Target

\subsection{Experimental Setup}

The experimental shaped charges whose model is in Fig. 4 after being filled with explosives and assembled are shown in Fig. 7. The experimental setup is shown in Fig. 8. The two-charge-diameter standoff distance is achieved by a plastic tube attached to the tip of the experimental shaped charge. 


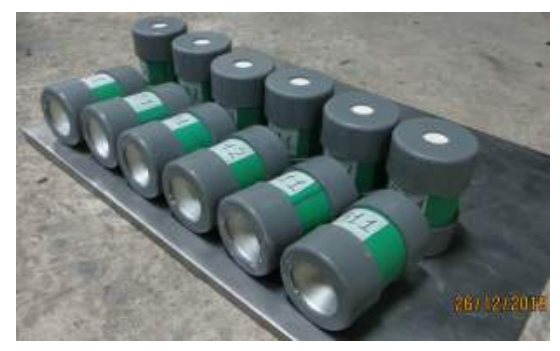

Fig. 7 Experimental shaped charges

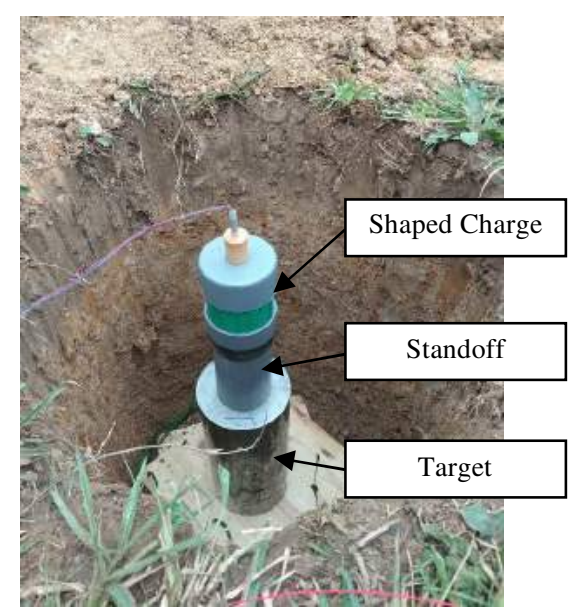

Fig. 8 Experimental setup

\subsection{Test Results of the Influence of Wave Shaper Position on Penetration Depth}

After the static explosion of the experimental samples, the steel targets were cut to determine the penetration depth (Fig. 9).

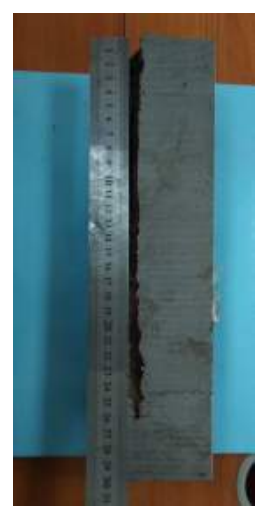

(a) $z w s=-33 \mathrm{~mm}$

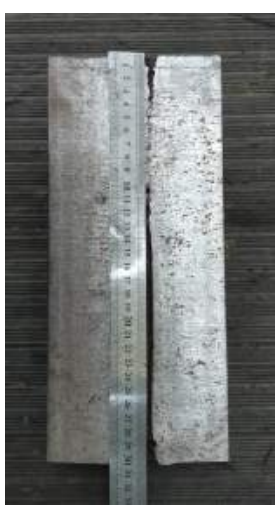

(b) $z w s=-23 \mathrm{~mm}$

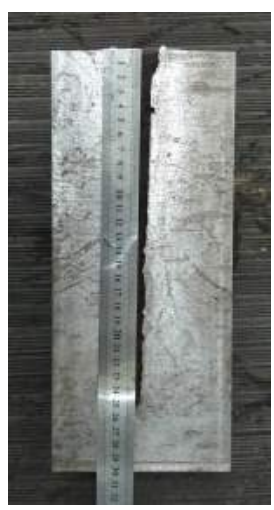

(c) $z w s=-13 \mathrm{~mm}$

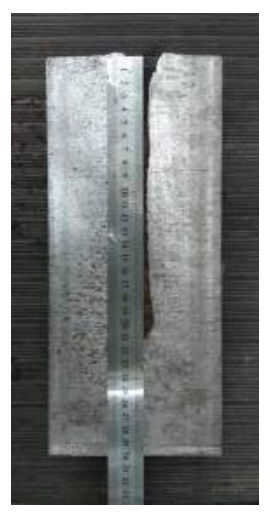

(d) $z w s=0 \mathrm{~mm}$

Fig. 9 A number of steel targets cut at different positions of the wave shaper

Experimental penetration depth results for each wave shaper position are shown in Tab. 3.

\section{Analysis and Discussion}

In this chapter, analyses of detonation wave propagation behavior and jet tip velocity will be presented. These analyses can explain the tendency of changing of simulational penetration, which was compared with experimental penetration. 


\subsection{Detonation Wave Propagation Behavior}

When the wave shaper is inserted in a shaped charge, it can change the direction of the detonation wave surface, which propagates in the charge. The detonation wave surface behind the wave shaper includes the interference of the wave surfaces. One part of the wave propagates around the wave shaper, and the rest passes through the wave shaper. If the angle between the detonation wave surface and liner generatrix is called $\beta$, the simulation results show that the angle $\beta$ decreases as the wave shaper position approaches the top of the liner (Fig. 10). According to [4], when the angle $\beta$ decreases, the detonation products behind the detonation wave surface will impact the liner at a higher speed, which increases the collapse velocity.

Tab. 3 Experimental penetration depth results

\begin{tabular}{|c|c|c|c|}
\hline$z w s[\mathrm{~mm}]$ & Shot & Penetration depth $[\mathrm{mm}]$ & Average penetration depth $[\mathrm{mm}]$ \\
\hline \multirow{3}{*}{-33} & 1 & 286 & \multirow{3}{*}{274} \\
\hline & 2 & 262 & \\
\hline & 3 & 275 & \\
\hline \multirow{3}{*}{-23} & 1 & 286 & \multirow{3}{*}{288} \\
\hline & 2 & 277 & \\
\hline & 3 & 300 & \\
\hline \multirow{3}{*}{-13} & 1 & 279 & \multirow{3}{*}{269} \\
\hline & 2 & 261 & \\
\hline & 3 & 267 & \\
\hline \multirow{3}{*}{0} & 1 & 209 & \multirow{3}{*}{219} \\
\hline & 2 & 239 & \\
\hline & 3 & 210 & \\
\hline
\end{tabular}

\subsection{Jet Tip Velocity and Jet Velocity Gradient}

When the wave shaper position is towards the top of the liner, the detonation wave surface changes in the direction of reducing the angle $\beta$. It causes an increase in the pressure of the wave surface. As a result, the collapse velocity increases. The liner elements that move towards the symmetry axis of the shaped charge form the jet tip with increasing tip velocity (Tab. 4). The jet tip velocity at $z w s=0 \mathrm{~mm}$ increased by $18.1 \%$ compared to the one at $z w s=-33 \mathrm{~mm}$. Also, the jet velocity gradient increases (Fig. 11). It is one of the factors that increase penetration depth.

The increase in the jet tip velocity and the jet velocity gradient causes the kinetic energy of the jet increase. However, as the wave shaper position moves closer to the top of the liner, the mass of the liner elements involved in the jet tip decrease. It is the reason for the reduction of jet kinetic energy despite the high jet tip velocity and jet velocity gradient. The jet kinetic energy at $z w s=-33 \mathrm{~mm}$ is $19.1 \%$ greater than the one at $z w s=0 \mathrm{~mm}$. The jet kinetic energy diagram according to the wave shaper position at a two-charge-diameter standoff distance is shown in Fig. 12.

\subsection{Penetration Performance}

The penetration depth results calculated by the simulation method (Fig. 13) show that, when the wave shaper position approaches the top of the liner, the penetration depth increases gradually and then decreases. The maximum value of penetration depth is $321 \mathrm{~mm}$ at the position $z w s=-23 \mathrm{~mm}$. 


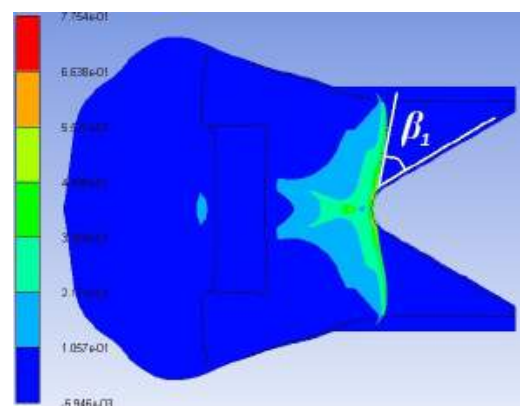

(a) $z w s=-33 \mathrm{~mm} ; t=11.5 \mu \mathrm{s}$

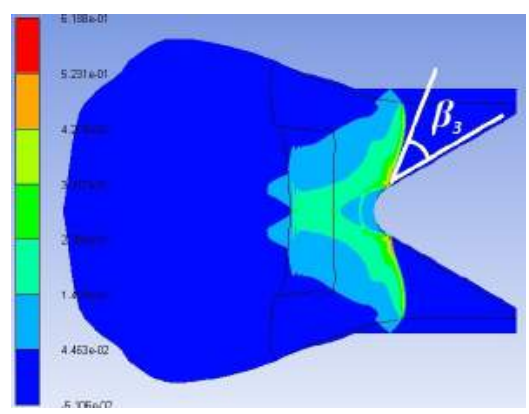

(c) $z w s=-13 \mathrm{~mm} ; t=11.4 \mu \mathrm{s}$

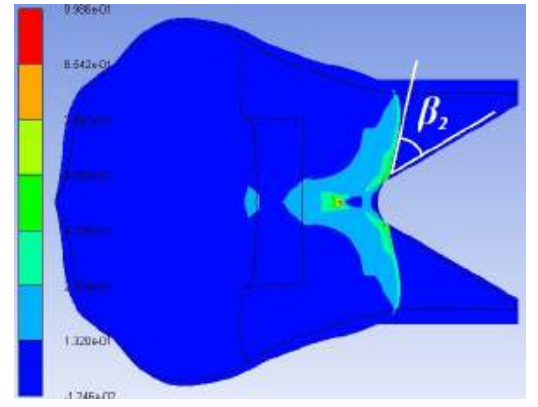

(b) $z w s=-23 \mathrm{~mm} ; t=11.45 \mu \mathrm{s}$

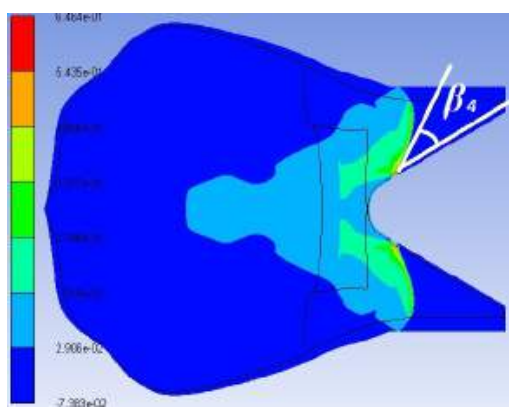

(d) $z w s=0 \mathrm{~mm} ; t=11.8 \mu \mathrm{s}$

Fig. 10 Propagation behavior of detonation wave in explosion process

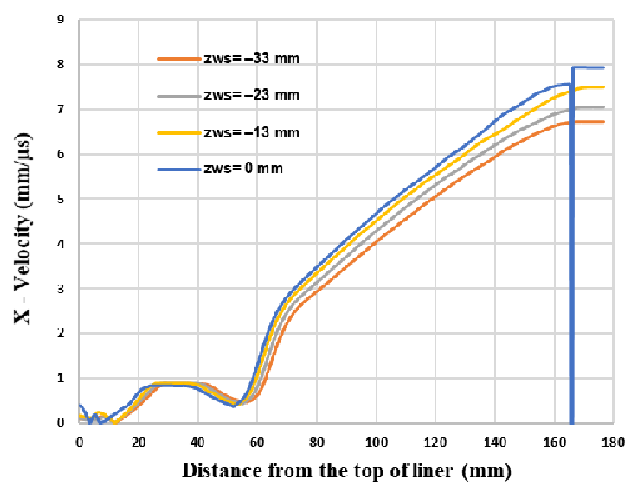

Fig. 11 Velocity gradient of jet along the symmetry axis of the shaped charge

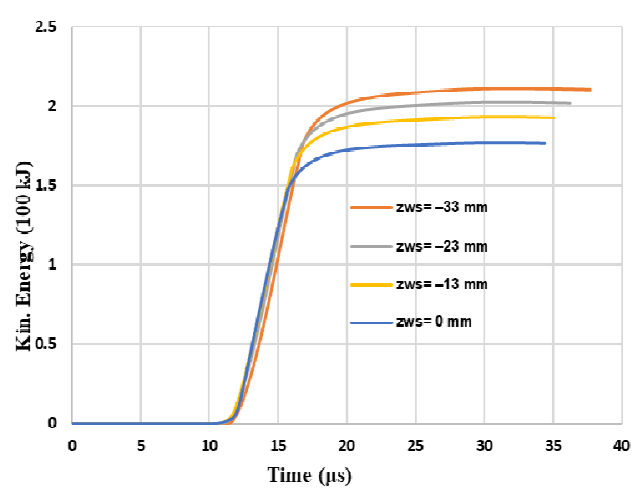

Fig. 12 Energy-time curves for different positions of the wave shaper

Tab. 4 Tip velocity according to different positions of the wave shaper at a two-charge-diameter

\begin{tabular}{|c|c|c|c|c|}
\hline$z w s[\mathrm{~mm}]$ & -33 & -23 & -13 & 0 \\
\hline Jet tip velocity $[\mathrm{mm} / \mu \mathrm{s}]$ & 6.719 & 7.048 & 7.505 & 7.935 \\
\hline
\end{tabular}




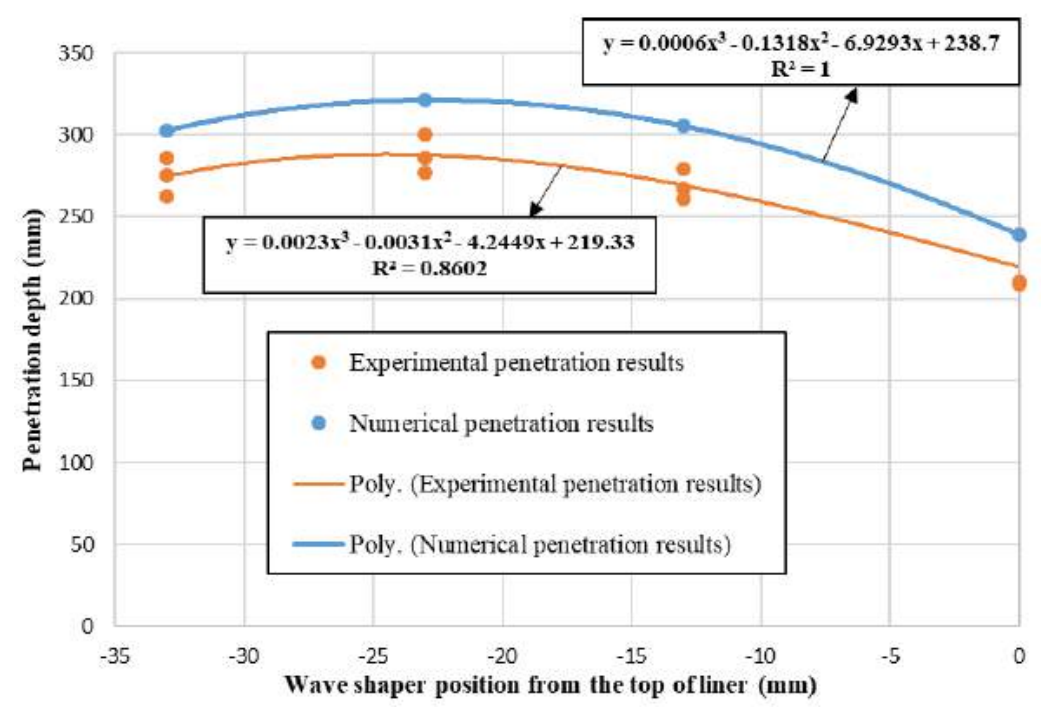

Fig. 13 Comparison of experimental and numerical penetration results

When zws increases from $-33 \mathrm{~mm}$ to $-23 \mathrm{~mm}$, the penetration depth increases. The increasing trend of the penetration depth is because the closer the wave shaper position approaches the top of the liner, the more perpendicularly the wave surface is compressed to the liner surface, and the smaller the angle $\beta$ is, respectively (Fig. 10). As a result, the jet tip velocity and jet velocity gradient increase. These factors are the cause of increasing penetration depth.

When zws continues to increase from $-23 \mathrm{~mm}$ to $0 \mathrm{~mm}$ (at the top of the liner), the penetration depth tends to decrease. This is explained by two reasons: one is the decrease in jet tip mass and the other is the increase in velocity gradient (Fig. 11). These factors cause the kinetic energy to decrease (Fig. 12) and the jet to be broken during the penetration. Therefore, the penetration depth decreases as the wave shaper position is closer to the top of the liner.

The deviations between the simulation and the experimental average results of the penetration depth with $z w s=-33 \mathrm{~mm}, z w s=-23 \mathrm{~mm}, z w s=-13 \mathrm{~mm}$ and $z w s=0 \mathrm{~mm}$ are $10.2 \%, 11.6 \%, 13.5 \%$ and $8.8 \%$, respectively. These results show the validity of the simulation method when the wave shaper position changes. The deviation between simulation and experiment is less than $15 \%$ within the permitted range.

\section{Conclusion}

The influence of the wave shaper position on the jet formation and the penetration depth to the steel target is presented in this study. The following conclusions have been drawn:

- when the wave shaper position approaches the top of the liner, the detonation wave surface changes in the direction of reducing the angle between the detonation wave surface and liner generatrix. This effect increases the pressure of the wave surface and causes an increase in jet tip velocity and velocity gradient, 
- when the wave shaper position is closer to the top of the liner, there is a value of the wave shaper position at which the penetration depth is maximized,

- the simulation method presented in this study allows assessing the influence of wave shaper position on the jet parameters and the penetration depth. The experimental results show the validity of the simulation method. The difference between the penetration depth of simulation and the experiment is less than $15 \%$.

\section{References}

[1] ANDREEV, S.G., BABKIN, A.V., BAUM, F.A., IMKHOVIK, N.A., KOBYLKIN, I.F., KOLPAKOV, V.I., LADOV, S.V., ODINTSOV, V.A., ORLENKO L.P., OKHITIN, V.N., SELIVANOV, V.V., SOLOVIEV, V.S., STANYUKOVICH, K.P., CHELYSHEV, V.P. and SHEKHTER, B.I. Physics of Explosion (in Russian). Moscow: Fizmatlit, 2002. ISBN 5-9221-0219-2.

[2] ZHU, C.S., HUANG, Z.X., ZU, X.D. and XIAO, Q.Q. Mach Wave Control in Explosively Formed Projectile Warhead. Propellants, Explosives, Pyrotechnics, 2014, vol. 39, no. 6, p. 909-915. DOI 10.1002/prep.201400106.

[3] ZU, X.D., HUANG, Z.X., ZHU, C.S. and XIAO, Q.Q. Study of Detonation Wave Contours in EFP Warhead. Defence Technology, 2016, vol. 12, no. 2, p. 129-133. DOI 10.1016/j.dt.2016.01.002.

[4] GUO, H.G., ZHENG, Y.F., TANG, L., YU, Q.B., GE, C. and WANG, H.F. Effect of Wave Shaper on Reactive Materials Jet Formation and Its Penetration Performance. Defence Technology, 2019, vol. 15, no. 4, p. 495-505. DOI 10.1016/j.dt.2019.05.005.

[5] NAEEM, K. and HUSSAIN, A. Numerical and Experimental Study of Wave Shaper Effects on Detonation Wave Front. Defence Technology, 2018, vol. 14, no. 1, p. 45-50. DOI 10.1016/j.dt.2017.11.005.

[6] GÜREL, E. Modeling and Simulation of Shaped Charges [on line]. [Master's Thesis]. Ankara: Middle East Technical University, 2009. 130 p. [viewed 201911-02]. Available from: https://etd.lib.metu.edu.tr/upload/12610830/index.pdf

[7] ELSHENAWY, T.A.E. Criteria of Design Improvement of Shaped Charges Used As Oil Well Perforators [on line]. [PhD Thesis]. Manchester: University of Manchester, 2012. 253 p. [viewed 2019-10-15]. Available from: https://www. escholar.manchester.ac.uk/uk-ac-man-scw:183508

[8] ANSYS Autodyn User's Manual [on line]. Canonsburg: SAS IP, 2013. 502 p. [viewed 2019-10-22]. Available from: https://vdocuments.net/ansys-autodynusers-manual.html

[9] URTIEW, P.A., VANDERSALL, K.S., TARVER, C.M., GARCIA, F. and FORBES, J.W. Shock Initiation Experiments and Modeling of Composition B And C-4. In Proceedings of the $13^{\text {th }}$ International Detonation Symposium. Norfolk: Lawrence Livermore National Laboratory, 2006, p. 929-939. DOI 10.1134/S1990793108020036.

[10] VAZIRI, M.R., SALIMI, M. and MASHAYEKHI, M. A New Calibration Method for Ductile Fracture Models as Chip Separation Criteria in Machining. Simulation Modelling Practice and Theory, 2010, vol. 18, no. 9, p. 1286-1296. DOI 10.1016/j.simpat.2010.05.003. 